\title{
Identification of urine neutrophil gelatinase-associated lipocalin molecular forms and their association with different urinary diseases in cats
}

\author{
Po-Han Wu ${ }^{1 \dagger}$, Wei-Li Hsu ${ }^{2 \dagger}$, Pei-Shiue Jason Tsai ${ }^{3}$, Vin-Cent Wu ${ }^{4}$, Han-Ju Tsai ${ }^{5}$ and Ya-Jane Lee ${ }^{1,5^{*}}$ (B)
}

\begin{abstract}
Background: Neutrophil gelatinase-associated lipocalin (NGAL), a promising renal biomarker, can exists as a monomer, a dimer and/or in a NGAL/matrix metalloproteinase-9 (MMP-9) complex form when associated with different urinary diseases in humans and dogs. In this study, the presence of the various different molecular forms of NGAL in cat urine (uNGAL) was examined and whether these forms are correlated with different urinary diseases was explored.

Results: One hundred and fifty-nine urine samples from cats with various different diseases, including acute kidney injury (AKI, 22 cats), chronic kidney disease (CKD, 55 cats), pyuria (44 cats) and other non-renal and non-pyuria diseases (non-RP, 26 cats), as well as healthy animals (12 cats), were collected. The molecular forms of and concentrations of urinary NGAL in these cats were analyzed, and their UNGAL-to-creatinine ratio (UNCR) were determined. The cats with AKI had the highest UNCR (median: $2.92 \times 10^{-6}$ ), which was followed by pyuria (median: $1.43 \times 10^{-6}$ ) and CKD (median: $0.56 \times 10^{-6}$ ); all of the above were significantly higher than the healthy controls (median: $\left.0.17 \times 10^{-6}\right)(p<0.05)$. Three different NGAL molecular forms as well as the MMP-9 monomer were able to be detected in the cat urine samples. Moreover, the cases where urine NGAL monomer were present also had significantly higher levels of BUN (median: 18.9 vs $9.6 \mathrm{mmol} / \mathrm{L}$ ) and creatinine (327.1 vs $168 \mathrm{umol} / \mathrm{L}$ ). The presence of dimeric NGAL was found to be associated with urinary tract infections. Most cats in the present study $(126 / 159,79.2 \%)$ and more than half of healthy cats $(7 / 12,58.3 \%)$ had detectable NGAL/MMP-9 complex present in their urine.
\end{abstract}

Conclusions: The monomeric and dimeric molecular forms of UNGAL suggest upper and lower urinary tract origins of disease, respectively, whereas the presence of the UNGAL/MMP-9 complex is able to be detected in most cats, including seemingly healthy ones.

Keywords: Molecular forms, Biomarker, NGAL, MMP-9, Feline urinary diseases

\section{Background}

Neutrophil gelatinase-associated lipocalin (NGAL), a 25 $\mathrm{kDa}$ glycoprotein belonging to lipocalin superfamily, was first purified from human neutrophils in the early 1990s $[1,2]$. In humans and dogs, NGAL has been reported to

\footnotetext{
* Correspondence: yajanelee@ntu.edu.tw

${ }^{\dagger}$ Po-Han Wu and Wei-Li Hsu contributed equally to this work.

${ }^{1}$ Institute of Veterinary Clinical Science, School of Veterinary Medicine, College of Bio-Resources and Agriculture, National Taiwan University, No. 153, Sec. 3, Keelung Rd, Taipei 106, Taiwan

${ }^{5}$ National Taiwan University Veterinary Hospital, College of Bio-Resources and Agriculture, National Taiwan University, No. 153, Sec. 3, Keelung Rd, Taipei 106, Taiwan

Full list of author information is available at the end of the article
}

serve as a biomarker for acute kidney injury (AKI) [3] and chronic kidney disease (CKD) [4-6]. In humans and dogs, NGAL is known exist in a number of forms, namely as $25 \mathrm{kDa}$ monomers, as $45 \mathrm{kDa}$ dimers, or in a $135 \mathrm{kDa}$ NGAL/ MMP9 complex form where it is covalently conjugated with matrix metalloproteinase-9 (MMP9) [1, 7]. Compared to monomeric MMP-9 in urine, the urine NGAL/ MMP9 complex is characterized by having a higher molecular weight, and this conjugation with NGAL might help to protect MMP-9 from degradation [8]. Monomeric NGAL and, to some extent the dimeric form, has been reported to be the predominant

(c) The Author(s). 2019 Open Access This article is distributed under the terms of the Creative Commons Attribution 4.0 International License (http://creativecommons.org/licenses/by/4.0/), which permits unrestricted use, distribution, and reproduction in any medium, provided you give appropriate credit to the original author(s) and the source, provide a link to the Creative Commons license, and indicate if changes were made. The Creative Commons Public Domain Dedication waiver (http://creativecommons.org/publicdomain/zero/1.0/) applies to the data made available in this article, unless otherwise stated. 
forms produced by renal tubular epithelial cells, whereas dimeric NGAL is believed to be mainly secreted by neutrophils in humans [9].

In veterinary medicine, NGAL has been proved to be a novel and promising biomarker. An increased level of NGAL in dogs has been found to be associated with various different urinary diseases [5, 10-13]. Among cats with naturally occurring kidney diseases, urine NGAL (uNGAL) levels and the urine NGAL to creatinine ratio (UNCR) are thought to be a useful biomarkers when detecting chronic renal damage and when predicting the clinical progression of cats with CKD [14]. However, the presence and the various levels of the different molecular forms of uNGAL have not yet been investigated in cats. The first aim of this study was to determine the presence and origin of the various different molecular forms of uNGAL in cats. The second aim was to investigate the correlation in cats between the different molecular forms of uNGAL, as well as the concentration of UNGAL, with various different urinary diseases.

\section{Results}

In total, samples of 159 cats, which were able to be classified into a number of distinct groups (Fig. 1), were enrolled in the present study. The UNGAL concentrations were measured by ELISA.

The uNGAL concentration values and the UNCR of all of the groups are presented in Table 1. Urine samples from the $\mathrm{A}+\mathrm{P}$ group had the highest level of UNGAL, which was followed by the AKI group, the pyuria group, the non-RP group, the healthy controls and finally the CKD group, in descending order. There were no significant differences in uNGAL levels among the $\mathrm{A}+\mathrm{P}$, AKI and pyuria groups. The uNGAL values of the $\mathrm{A}+\mathrm{P}$ and AKI groups were significantly higher than those of the non-RP, control and CKD groups. The concentration of uNGAL obtained for the non-RP group was significantly higher than the values for the control and CKD groups. After adjusting the absolute value of uNGAL based on the urine creatinine concentration, the levels of UNCR were determined and compared among groups. The values for UNCR in descending order were the $\mathrm{A}+\mathrm{P}$ group, the AKI group, the pyuria group, the CKD group, the non-RP group and the healthy control cats. The $\mathrm{UNCR}$ values of the $\mathrm{A}+\mathrm{P}$ and AKI groups were significantly higher than the values for the CKD group, the non-RP group and the healthy controls. Significant differences in UNCR were found between the A + P group and the pyuria group, and between the AKI group and the CKD group, but no significant difference was found between the AKI group and the pyuria group.

The molecular forms of the two target proteins in all of the enrolled urine samples were analyzed by Western blot analysis using rabbit anti-canine NGAL antibodies. Under non-reducing condition, three different molecular forms of uNGAL were successfully detected in the feline samples, namely the $27-\mathrm{kDa}$ monomer, the $48-\mathrm{kDa}$ dimer and the 140-kDa NGAL/MMP-9 complex (Fig. 2a). The existence of the 140-kDa NGAL/MMP-9 complex in feline urine was further confirmed by Western blot

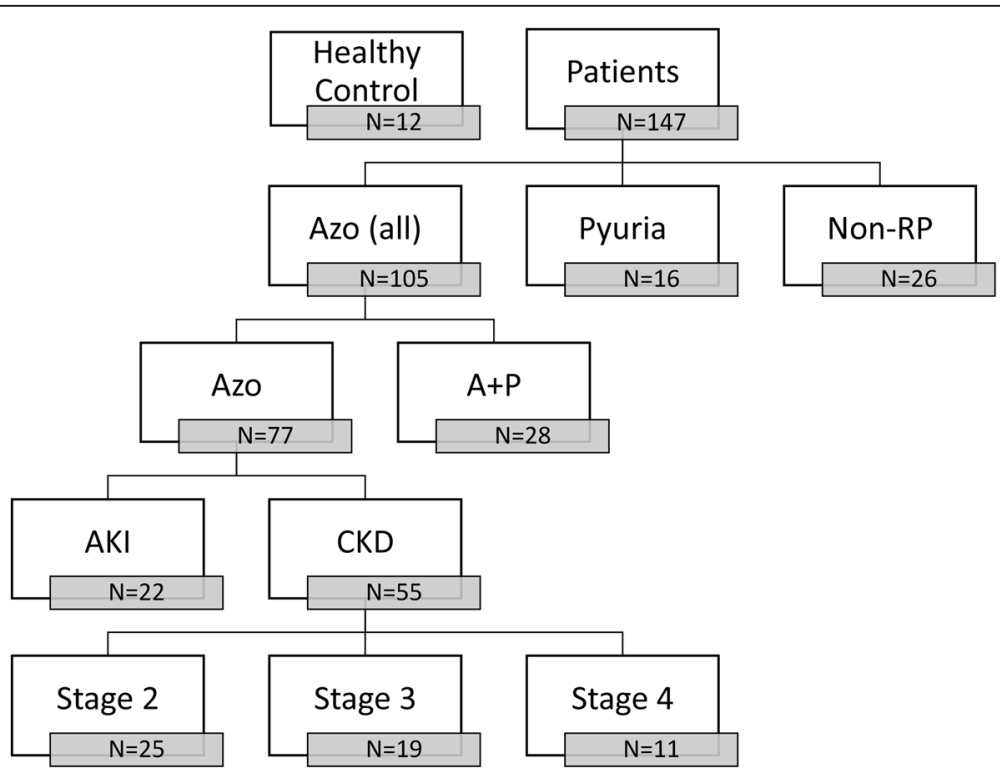

Fig. 1 Diagram of the case grouping in this study. The Azo (all) group includes the cats with azotemia; the Azo group consists of the azotemia cats without pyuria, while the A + P group includes the cats having both pyuria and azotemia. The non-RP group consists of the cats with diseases unrelated to azotemia and pyuria; while the AKI group is made up of cats with acute kidney disease. The CKD group consists of cats with chronic kidney disease 


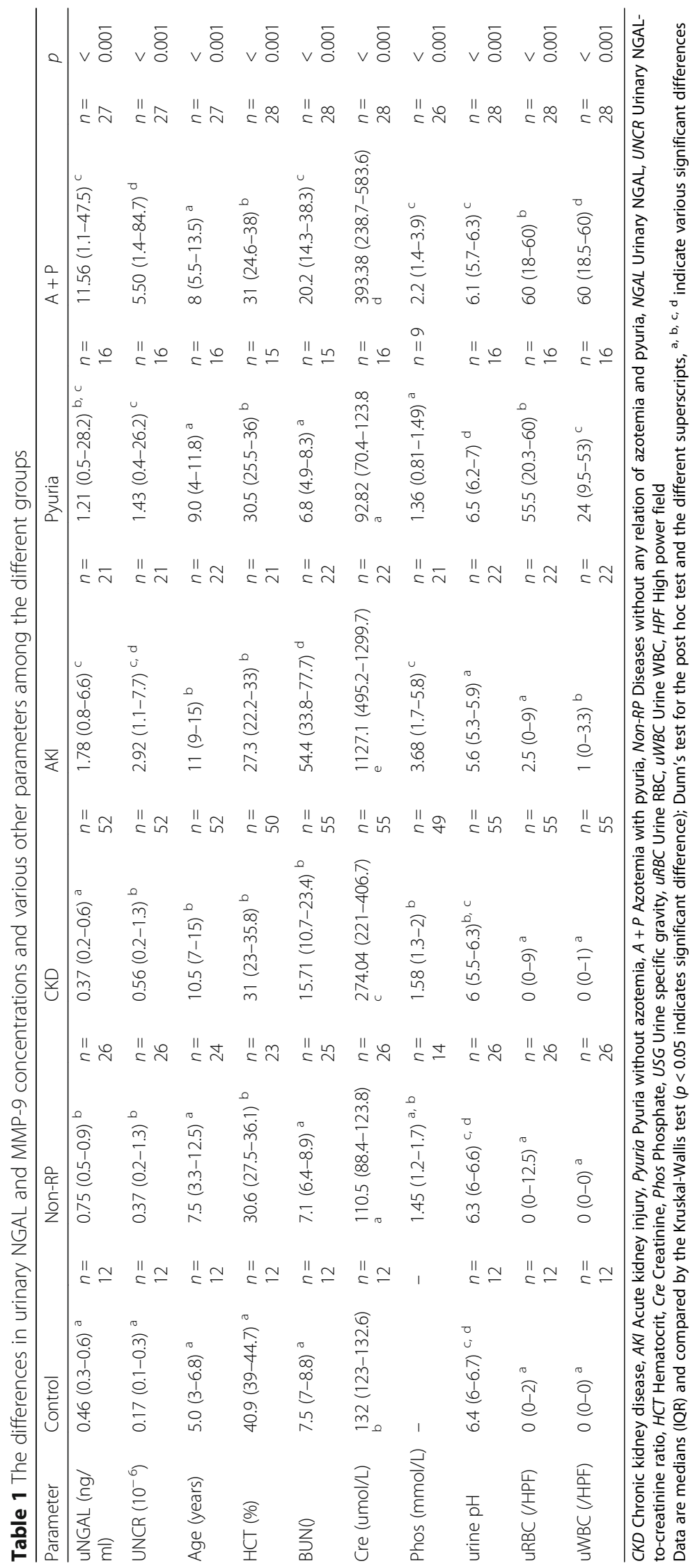




\section{A NGAL}

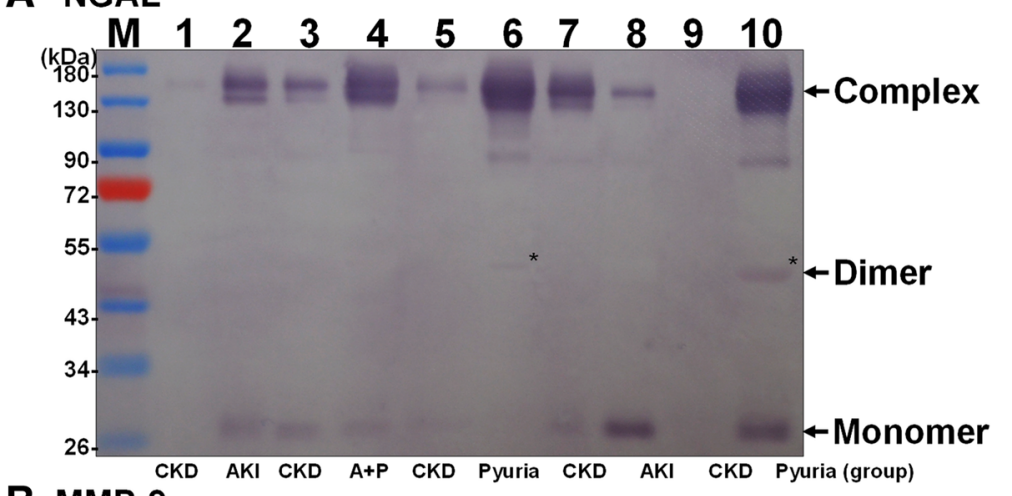

B MMP-9

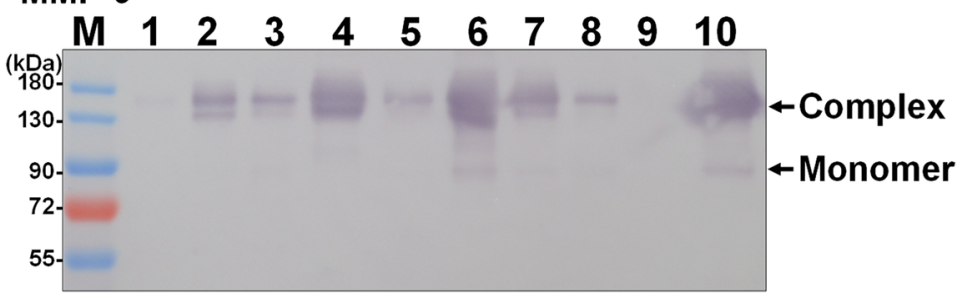

Fig. 2 The molecular forms of NGAL and MMP 9 present in feline urine samples. The proteins in the urine samples from representative cats with CKD (lane 1, 3, 5, 7, 9), AKI (lane 2, 8), A + P (land 4), and pyuria (lane 6, 10) were separated by non-reducing SDS-PAGE. The presence of NGAL and MMP9 was detected by Western blot analysis using antibodies specific for NGAL (a) and MMP-9 (b). The protein complex with a molecular weight of $140-\mathrm{kDa}$ was simultaneously detected by both antibodies, anti-NGAL (a) and anti-MMP-9 (b), and was therefore identified as the NGAL/MMP-9 complex

analysis using rabbit anti-canine MMP-9 antibodies. In addition, the $90-\mathrm{kDa}$ MMP-9 monomer was also successfully identified in our feline urine samples (Fig. 2b).

Overall, uNGAL monomer was detected in 74 cats $(48.1 \%, 74 / 154)$. Compared to the cats without uNGAL monomer, the cats with monomeric uNGAL on urinalysis were characterized as having a significantly higher level of uNGAL, a significantly higher level of UNCR, a lighter body weight, a lower level of HCT, a higher WBC count, a higher segmented neutrophil count, a higher BUN value, a higher creatinine value, a higher phosphorus in blood value, a higher level of UPC and a lower $\mathrm{pH}$ values (Table 2 ).

The presence of dimeric UNGAL was noted in 44 cats (27.7\%, 44/159). The cats with dimeric UNGAL had significantly higher levels of uNGAL and UNCR. The significantly different parameters between the cats with and without dimeric UNGAL were a higher segmented

Table 2 The differences in various variables between the groups with or without UNGAL monomer present in the urine samples

\begin{tabular}{|c|c|c|c|c|c|}
\hline Parameter & uNGAL Monomer (-) 52.2\% (83/159) & & $\begin{array}{l}\text { uNGAL Monomer }(+) \\
47.8 \%(76 / 159)\end{array}$ & & $p$ \\
\hline UNGAL (ng/ml) & $0.5(0.0 .3-1.3)$ & $n=80$ & $0.9(0.5-2.9)$ & $n=74$ & 0.006 \\
\hline UNCR $\left(10^{-6}\right)$ & $0.4(0.2-1.9)$ & $n=80$ & $1.3(0.5-4.8)$ & $n=74$ & $<0.001$ \\
\hline Seg. $(10 \wedge 9 / L)$ & $6.1(3.8-9.2)$ & $n=72$ & $12.8(6.8-20.8)$ & $n=75$ & $<0.001$ \\
\hline BUN (mmol/L) & $9.6(7.5-16.4)$ & $n=81$ & $18.92(8.9-47.5)$ & $n=76$ & $<0.001$ \\
\hline Creatinine (umol/L) & $168(132.6-282.9)$ & $n=83$ & $327.1(132.6-663.2)$ & $n=76$ & 0.002 \\
\hline Phosphorus (mmol/L) & $1.4(1.2-2)$ & $n=56$ & $1.9(1.5-3.8)$ & $n=65$ & $<0.001$ \\
\hline urine $\mathrm{pH}$ & $6.2(5.8-6.5)$ & $n=83$ & $5.9(5.5-6.3)$ & $n=76$ & $<0.001$ \\
\hline UPC & $0.1(0-0.45)$ & $n=30$ & $0.7(0.4-1.9)$ & $n=29$ & $<0.001$ \\
\hline uRBC (/HPF) & $0(0-21)$ & $n=83$ & $2.5(0-58)$ & $n=76$ & 0.127 \\
\hline uWBC (/HPF) & $0(0-8)$ & $n=83$ & $1(0-9)$ & $n=76$ & 0.240 \\
\hline
\end{tabular}

uNGAL Urinary NGAL, UNCR Urinary NGAL-to-creatinine ratio, WBC White blood cells, Seg. Segmented neutrophil, BUN Blood urea nitrogen, UPC Urinary protein-tocreatinine ratio, UWBC Urine white blood cells, URBC Urine red blood cell, HPF High power field

Data are medians (IQR) and compared by the Mann-Whitney $U$-test $(p<0.05$ indicates significant difference) 
Table 3 The differences in various variables between the group with or without NGAL dimer present in the urine

\begin{tabular}{|c|c|c|c|c|c|}
\hline Parameter & $\begin{array}{l}\text { uNGAL Dimer (-) } \\
72.3 \%(115 / 159)\end{array}$ & & $\begin{array}{l}\text { uNGAL Dimer (+) } \\
27.7 \%(44 / 159)\end{array}$ & & $p$ \\
\hline uNGAL (ng/ml) & $0.6(0.3-1.2)$ & $n=111$ & $2.3(0.6-47.5)$ & $n=43$ & $<0.001$ \\
\hline $\operatorname{UNCR}\left(10^{-6}\right)$ & $0.6(0.2-1.9)$ & $n=111$ & $3.4(0.5-53.7)$ & $n=43$ & $<0.001$ \\
\hline Seg. $(10 \wedge 9 / L)$ & $7.3(4.3-14.3)$ & $n=106$ & $11.1(6.9-18.5)$ & $n=41$ & 0.010 \\
\hline BUN (mg/dL) & $33(21-74)$ & $n=114$ & $34(21-78)$ & $n=43$ & 0.937 \\
\hline Creatinine (mg/dL) & $2.6(1.5-5.6)$ & $n=115$ & $2.2(1.2-4.5)$ & $n=44$ & 0.124 \\
\hline Phosphorus (mg/dL) & $5.0(4.2-8.9)$ & $n=86$ & $5.1(4.2-10)$ & $n=35$ & 0.975 \\
\hline urine $\mathrm{pH}$ & $6(5.6-6.4)$ & $n=115$ & $6.1(5.7-6.4)$ & $n=44$ & 0.543 \\
\hline UPC & $0.3(0.1-0.9)$ & $n=47$ & $0.5(0.1-1.7)$ & $n=12$ & 0.491 \\
\hline URBC (/HPF) & $0(0-20)$ & $n=115$ & $13(0-60)$ & $n=44$ & $<0.001$ \\
\hline UWBC (/HPF) & $0(0-2)$ & $n=115$ & $14(2-60)$ & $n=44$ & $<0.001$ \\
\hline
\end{tabular}

uNGAL Urinary NGAL, UNCR Urinary NGAL-to-creatinine ratio, WBC White blood cells, Seg. Segmented neutrophil, BUN Blood urea nitrogen, UPC Urinary protein-tocreatinine ratio, UWBC Urine white blood cells, URBC Urine red blood cell, HPF High power field

Data are medians (IQR) and compared by the Mann-Whitney U-test

neutrophil count in blood, a higher level of urinary protein, a higher urine RBC count and a higher urine WBC count. (Table 3).

In total, the NGAL/MMP-9 complex was present in urine of 126 cats $(126 / 159,79.2 \%)$. Furthermore, over half of the cats in healthy control group also had NGAL/ MMP-9 complex present in their urine (7/12, 58.3\%). No parameters were found to be significantly different between the cats with and without NGAL/MMP-9 complex across of the groups studied here.

Among the CKD group $(n=55)$, there were 25 cats in stage 2,19 cats in stage 3 and 11 cats in stage 4 . The occurrence frequencies of urinary monomeric NGAL, the NGAL/MMP-9 complex and monomeric MMP-9 were all in ascending order starting with the control group as lowest and increasing with stage. The proportion of cats with urinary NGAL monomer present was significantly higher for CKD stage 3 cats and CKD stage 4 cats compared to the control group cats and CKD stage 2 cats (both $p<0.001$ for versus control group; both $p<0.05$ versus CKD stage 2). (Table 4).
At the time of sample collection, 56 urine samples were subjected to the urine culture. According the results of these urine cultures, these 56 cats could be divided into a UTI $(-)$ group $(n=38)$ and a UTI $(+)$ group $(n=18)$. A comparison between these two groups indicated that the presence of dimeric NGAL was significantly higher in the cats with positive urine culture results (Table 5). Both UNGAL and UNCR were significantly correlated independently with the level of serum creatinine and the severity of pyuria (uWBC) (Table 6).

\section{Discussion}

To our knowledge, this is the first study to evaluate the presence of the different molecular forms of uNGAL in cats. The various molecular forms of UNGAL and MMP-9 in cats were able to be successfully detected by Western blot analysis using anti-canine NGAL and anticanine MMP-9 antibodies. Similar to previous findings in human patients [9] and dogs [7], three different forms of NGAL were found to be present in the feline urine samples, these were the $27 \mathrm{kDa}$ monomer, the $48 \mathrm{kDa}$

Table 4 The differences of urine NGAL and MMP-9 between the control and CKD subgroups

\begin{tabular}{|c|c|c|c|c|c|}
\hline Parameter & $\begin{array}{l}\text { Control } \\
(n=12)\end{array}$ & $\begin{array}{l}\text { Stage } 2 \\
(n=25)\end{array}$ & $\begin{array}{l}\text { Stage } 3 \\
(n=19)\end{array}$ & $\begin{array}{l}\text { Stage } 4 \\
(n=11)\end{array}$ & $p$ \\
\hline uNGAL (ng/ml) ${ }^{d}$ & $0.5(0.3-0.6)^{a, b} n=12$ & $\begin{array}{l}0.3(0.2-0.5)^{a} \\
n=23\end{array}$ & $\begin{array}{l}0.5(0.3-0.9)^{b} \\
n=18\end{array}$ & $\begin{array}{l}0.6(0.3-1.2)^{b} \\
n=11\end{array}$ & $0.030^{*}$ \\
\hline UNCR $\left(10^{-6}\right)^{d}$ & $\begin{array}{l}0.17(0.1-0.3)^{a} \\
n=12\end{array}$ & $\begin{array}{l}0.17(0.1-0.5)^{a} \\
n=23\end{array}$ & $\begin{array}{l}0.605(0.3-1.4)^{b} \\
n=18\end{array}$ & $\begin{array}{l}1.33(0.9-2.7)^{c} \\
n=11\end{array}$ & $<0.001^{* *}$ \\
\hline NGAL Monomer e & $0 \%(0 / 12)$ & $20 \%(5 / 25)$ & $63.2 \%(12 / 19)$ & $81.8 \%(9 / 11)$ & $<0.001^{* *}$ \\
\hline NGAL Dimer ${ }^{e}$ & $0 \%(0 / 12)$ & $20 \%(5 / 25)$ & 0\% (0/19) & $0 \%(0 / 11)$ & 0.643 \\
\hline NGAL/MMP-9 e & $58.3 \%(7 / 12)$ & $60 \%(15 / 25)$ & $84.2 \%(16 / 19)$ & $90.9 \%(10 / 11)$ & 0.102 \\
\hline
\end{tabular}

The different superscripts $\left({ }^{a}, b, c\right)$ indicate various significant differences

${ }^{\mathrm{d} D a t a}$ are medians (IQR) and compared by the Kruskal-Wallis test ( $p<0.05$ indicated significant difference) and post hoc by Dunn's test.

eFisher's exact test are used to compare the category data and presented in\%.

UNGAL Urine NGAL, UNCR Urine NGAL-to-creatinine ratio 
Table 5 The differences in various variables between UTI and non-UTI

\begin{tabular}{|c|c|c|c|c|c|}
\hline Parameter & UTI $(-)(n=38$ & & UTI $(+)(n=18)$ & & $p$ \\
\hline$\overline{\mathrm{UNGAL}}(\mathrm{ng} / \mathrm{ml})^{\mathrm{a}}$ & $0.8(0.4-1.7)$ & $n=37$ & $47.4(6.1-48.2)$ & $n=18$ & $<0.001^{*}$ \\
\hline UNCR $\left(10^{-6}\right)^{a}$ & $0.93(0.5-2)$ & $n=37$ & $41.77(11-86)$ & $n=18$ & $<0.001^{*}$ \\
\hline NGAL Monomer $(+)^{b}$ & $60.5 \%$ & $(23 / 38)$ & $50 . \%$ & $(9 / 18)$ & 0.457 \\
\hline $\operatorname{NGAL}$ Dimer $(+)^{c}$ & $34.8 \%$ & $(8 / 38)$ & $83.3 \%$ & $(15 / 18)$ & $<0.001^{*}$ \\
\hline NGAL/MMP-9 $(+)^{b}$ & $86.8 \%$ & $(33 / 38)$ & $83.3 \%$ & $(15 / 18)$ & 0.703 \\
\hline
\end{tabular}

UTI Urinary tract infection

${ }^{a}$ Data are medians (IQR) and tested by Mann-Whitney $\mathrm{U}$ test

${ }^{b}$ Data are analyzed by the Chi-square test

${ }^{c}$ Data are analyzed by the Fisher's exact test

$p<0.05^{*}$ indicates a significant difference

dimer and the $140 \mathrm{kDa}$ NGAL/MMP-9 complex forms. Additionally, the 90-kDa MMP-9 monomer was also successfully identified in our feline urine samples.

The results demonstrate that the appearance of NGAL monomer and MMP-9 monomer are both significantly associated with elevated levels of serum BUN, creatinine and phosphate, which are known to be linked with renal disease. These findings suggest that one or other of these or possibly both of these monomers may be associated with kidney injury in cats. Both UNGAL and UNCR have been reported to be promising renal biomarkers in cats with kidney disease [14]. Moreover, the UNCR of cats with azotemia is significantly higher than the values obtained from healthy control cats.

The comparison between the AKI and CKD groups revealed that cats with AKI have a significantly higher level of UNGAL and UNCR, as well as a higher proportion of NGAL monomer compared to CKD cats. These findings are similar to those presented in previous reports targeting humans [15] and dogs [10]. The NGAL monomer has been considered to originate from injured renal tubular epithelial cells [9]. Therefore, the higher proportion of cats with NGAL monomer in the AKI group than in the CKD group seems to reflect the severity of the renal tubular injury during feline AKI. However, the influence of glomerular or renal hemodynamic dysfunction cannot be accessed and further investigation was needed.

Surprisingly, the NGAL/MMP-9 complex was found to be present in the urine of the majority of cats enrolled

Table 6 The spearman's correlations between UNGAL, UNCR, and plasma creatinine, urine WBC

\begin{tabular}{lll}
\hline Parameters & $r$ & $p$ \\
\hline UNGAL and Creatinine & 0.213 & 0.008 \\
UNCR and Creatinine & 0.406 & $<0.001$ \\
UNGAL and UWBC & 0.474 & $<0.001$ \\
UNCR and UWBC & 0.474 & $<0.001$ \\
\hline
\end{tabular}

Creatinine: plasma creatinine, uWBC Urine WBC, uNGAL Urine NGAL UNCR Urine NGAL-to-creatinine ratio, $r$ in the present study $(126 / 159,79.2 \%)$. This included more than half of the cats in the healthy control group (7/12, 58.3\%). This finding is different from previous findings in dogs [7] and humans [9]. The presence of the NGAL/MMP-9 complex in the urine of normal cats may indicate that the NGAL/MMP-9 complex may play a role in the normal physiology of the cat urinary system or, on the other hand, that the healthy control cats had already some abnormalities that could not be detected by the traditional examination. This area is well worth further investigation.

In dogs, the presence of WBCs in urine is correlated with an increase in urine NGAL level $[7,16]$. Additionally, the presence of dimeric UNGAL has been found to be related to WBC counts in canine urine samples [7]. Consistently, the results of the present study reveal that the UNGAL and UNCR of cats in the pyuria group are both significantly higher than of the values for the healthy control cats. Moreover, the presence of dimeric NGAL was found to be significantly associated with the urine WBC counts in cats. The proportion of cats with dimeric UNGAL is significantly higher in the pyuria group than in the healthy control group. In this study, the pyuria group consists of cats without azotemia, but with other diseases. These findings indirectly demonstrate that the feline monomer UNGAL seems to originate from the kidneys, and feline dimeric UNGAL appears to originate from inflammation of lower urinary tract. Increased uNGAL has been reported to be associated with neoplasia and endotoxaemia [13]. In this study, monomeric NGAL is the predominant molecular form in the urine of the cats that form the non-RP group (data not shown). However, the renal function of the cats in the non-RP group was only evaluated using BUN, creatinine and urinary analysis, and thus early injury to the kidneys cannot be excluded. Additionally, various neoplastic diseases and/or septic illnesses also cannot be ruled out.

The proportion of cats with UNGAL monomer and concentrations of UNGAL and UNCR that were significantly higher were only present in cats with either CKD 
IRIS stage 3 or stage 4 , as compared to either the control group or CKD IRIS stage 2 group. These results suggest that these variables may be useful as progression factors [14] in relation to late stage CKD.

When the different molecular forms of these proteins were compared between cats with and without UTI, the results indicated that dimeric uNGAL exists as the predominate molecular form in the urine of cats with UTI. This finding suggests that the presence of UTI should be considered when interpreting urine NGAL values when feline renal disease is present. Moreover, it is likely the dimeric UNGAL might be derived from neutrophils [9], and thus might play a role in diseases where bacteria are present in feline urine.

There are several limitations in this study. First, urine bacterial culture should be the gold standard when diagnosing UTI; nevertheless not all of the cats in the present study underwent urine bacterial culture and this means that the UTI case numbers might be an underestimate. Under such circumstances, any elevation of uNGAL might be with the result of an undetected UTI. Second, feline idiopathic cystitis is thought to be an important lower urinary disease that involves cystic inflammation, but the design of this study means that we were unable to investigate the role of dimeric uNGAL in this disease. Third, although we tried to group the cases in a simple manner, the cats from which the clinical samples were obtained may in some cases have complicated urinary diseases. This means that our study does not provide an animal model approach that would be able to explore directly the role of each specific uNGAL molecular form in a given disease.

\section{Conclusions}

To date, an ELISA kit that allows the differential detection of the various molecular forms of NGAL is not available. To comprehensively understand the presence of these NGAL forms in feline urine, Western blot analysis remains the ideal system for the simultaneous differentiation of the various molecular forms of uNGAL based on their molecular weights. As with humans and dogs, the presence of the uNGAL monomer in cats seems to be correlated with renal injury, whereas the presence of dimeric UNGAL appears to be involved in pyuria and UTI. Nevertheless, unlike humans and dogs, the uNGAL/MMP-9 complex is able to be detected in most cats, even healthy ones.

\section{Methods}

\section{Cats and sample collection}

Feline urine samples were prospectively obtained from the cats that were admitted to National Taiwan University Veterinary Hospital, Taipei, Taiwan as part of feline routine diagnosis (IACUC Approval No: NTU 103-EL-
00084). Most feline urine samples were collected by cystocentesis $(73.6 \%, 117 / 159)$. However, samples were also obtained by catheterization (5.7\%, 9/159), voiding (15.1\%, 24/159), or aspiration using a subcutaneous ureteral bypass (SUB) device $(1.3 \%, 2 / 159)$ and these samples were also enrolled. Finally, there were seven urine samples $(4.4 \%, 7 / 159)$ that had an unrecorded urine collection method. The fresh urine samples were clarified by centrifugation at $1500 \mathrm{rpm}$ for $5 \mathrm{~min}$; the time between collection and centrifugation was limited within $30 \mathrm{~min}$. The urine supernatants were stored in microcentrifuge tubes at $-80^{\circ} \mathrm{C}$ until NGAL analysis. Important clinical information about each case, including history, the results of any physical examination and the results related to hematology, serum/plasma biochemistry and urinalysis were recorded for each cat. These parameters, including the number of segmented neutrophils in the blood, serum BUN, creatinine, phosphorus and urine protein-to-creatinine ratio (UPC), $\mathrm{uWBC}$, and $\mathrm{URBC}$, all of which have been reported to be associated with UNGAL, were used for the comparisons between the groups with and without the specific molecular forms of uNGAL. Blood chemistry, the urine protein/creatinine ratios and the complete blood counts were measured using a Vitros 350 chemistry system (Ortho clinical diagnostics), an IDEXX Catalyst Dx* Analyzer (IDEXX laboratories, Inc.), and an Exigo, (Boul Medical AB) respectively.

\section{Case grouping criteria}

The urine samples were classified into a number of different groups (Fig. 1). Cats without any clinical signs associated with any disease, with unremarkable physical examination findings and that had normal values for hematocrit (HCT), white blood cells (WBC), segmented neutrophils, alanine aminotransferase, aspartate transaminase, alkaline phosphatase, albumin, total protein, glucose, blood urea nitrogen (BUN), and creatinine, as well as the absence of any abnormalities on urinalysis, were classified as healthy control animals $(\mathrm{BUN}<10.7$ $\mathrm{mmol} / \mathrm{L}$, creatinine $<140 \mathrm{umol} / \mathrm{L}$, WBC in urine sediment $<5$ /high power field (HPF). The healthy cats were mainly cats belonging to students and colleagues, although some were cats that had been brought in to be neutered by surgery.

Cats with azotemia (creatinine $>140 \mathrm{umol} / \mathrm{L}$ ), and with a history and clinical signs that supported renal disease, were enrolled into the azotemia group. Based on the results of urinalysis, these azotemic cats were further divided into an azotemia-pyuria group (with $>5 \mathrm{WBCs}$ / HPF in sediment) and an azotemia group. However, cats with prerenal azotemia, where the azotemia was resolved after fluid therapy within $24 \mathrm{~h}$, were excluded. 
The cats with persistent azotemia (creatinine $>140$ umol/L longer than 1 month of duration) and with one of the clinical findings associated with CKD (e.g. polyuria, polydipsia, urine specific gravity $<1.030$, one or more small irregular kidneys (defined as one having a renal size of less than two times the length of the second lumber vertebrae by ventral dorsal view radiography or with decreased corticomedullary distinction by ultrasonography)) were included in the CKD group. The azotemia-CKD group was further classified into three subgroups based on the International Renal Interest Society (IRIS) staging system, these were (a) IRIS CKD stage II, (b) IRIS CKD stage III, (c) IRIS CKD stage IV. Cats with an acute onset of clinical signs shorter than 7 days (including those with oliguria, anuria, glucosuria, urinary cast, and enlarged kidneys), or with a plasma creatinine concentration increase of $\geq 26.5 \mathrm{umol} / \mathrm{L}$ from baseline within $48 \mathrm{~h}$, were defined as the AKI group. The cats without azotemia (creatinine $<140 \mathrm{umol} / \mathrm{L}$ ) and with $>5 \mathrm{WBCs} / \mathrm{HPF}$ on urine sediment examination were categorized into the pyuria group.

Cats with a normal plasma creatinine concentration (values < $140 \mathrm{umol} / \mathrm{L}$ ), and with $<5 \mathrm{WBCs} / \mathrm{HPF}$ in urine sediment, but with other diseases, were classified into the non-renal and non-pyuria (non-RP) group; this consisted of eight cases with neurological diseases, three cases with an infectious disease, three cases with a hepatic disease, two cases with a gastrointestinal disease, one case of aortic thromboembolism, one case of aplastic anemia, one case of pyometra and mammary gland tumor, one case of pyoperitoneum, one case of diabetes mellitus, one case of pancreatitis, one case of mast cell tumor, one case of hiatal hernia, one case of vaccine-associated sarcoma, and one case of lingual disease.

\section{Western blot analysis}

Urine samples were 4:1 diluted with premixed Laemmli protein sample buffer (4x Laemmli Sample Buffer, BioRad Laboratories Inc.) before the proteins were separated by non-reducing sodium dodecyl sulfate polyacrylamide gel electrophoresis (SDS-PAGE). The protein mix was directly loaded onto the gel without boiling. Subsequently, after SDS-PAGE, the gels were electrophoretically transferred to polyvinylidene difluoride (PVDF) membranes. The Western blot analysis followed the procedures that were described in a previous report [7]. The sequences of the dog and cat MMP-9s (Dog: NM_001003219.2; cat; XM_0039834612.4) shares 93\% similarity and therefore initially both canine and feline MMP-9 proteins were simultaneously detected by antibody against canine MMP-9 [7]. This confirmed that the canine-MMP-9 antibody was able to bind to MMP-9 proteins from both species; for that point onwards, canine-MMP-9 antibody was usedto detect the feline protein.
Briefly, for Western blotting, the membrane was blocked with phosphate buffered saline (PBS) containing 0.1\% Tween-20 (PBST) and 5\% dried skimmed milk for $1 \mathrm{~h}$ at room temperature and this was followed by incubation with a 1000-fold dilution of rabbit anti-canine antibodies against NGAL or MMP-9 overnight at $4{ }^{\circ} \mathrm{C}$. After washing with PBST three times, the membrane was incubated with a 10,000-fold dilution of secondary alkaline phosphatase (AP) conjugated anti-rabbit antibody for $1 \mathrm{~h}$ at room temperature. After further washes with PBST to remove the unbound antibodies, the signal on membrane was developed using 5-bromo-4-chloro-3indolyl phosphate (BCIP)/nitro blue tetrazolium (NBT) solutions (AP Conjugate Substrate Kit, Bio-Rad Laboratories Inc.) in the dark for $15 \mathrm{~min}$, and the image was recorded by digital camera.

Based on the expression pattern of the molecular forms of NGAL and MMP-9, all the cases were classified into the following categories: NGAL monomer $(+)$ and NGAL monomer (-) groups; NGAL dimer (+) and NGAL dimer (-) groups; and NGAL/MMP-9 complex $(+)$ and NGAL/MMP-9 complex (-) groups.

\section{Measurement of urine NGAL concentrations by ELISA}

Sandwich enzyme-linked immunosorbent assays (ELISAs) were conducted using the procedures described in a previous study [14]. Briefly, the capture anti-NGAL mouse polyclonal antibody was diluted 1: 800 fold in coating buffer and then was added to each well of an ELISA plate (Nunc TM). After blocking, 2-fold diluted urine samples and serially diluted feline recombinant NGAL calibration samples (with concentrations ranging from $17 \mathrm{ng} / \mathrm{mL}$ to $17 \mathrm{pg} / \mathrm{mL}$ and $0 \mathrm{pg} / \mathrm{mL}$ ) were individually added to each well and incubated at $4{ }^{\circ} \mathrm{C}$ for 16 h. Each experiment was carried out in duplicate. After washing with PBST three times, each well was loaded with $100 \mu \mathrm{l}$ of 2000 -fold diluted anti-NGAL rabbit polyclonal antibodies for detection and this was followed by incubation at $37^{\circ} \mathrm{C}$ for $2.5 \mathrm{~h}$. Subsequently, a 10,000-fold dilution of horseradish peroxidase (HRP) conjugated anti-rabbit secondary antibody was added to each well. After $1 \mathrm{~h}$ incubation, the plates were washed and TMB substrate (Tetramethylbenzidine, Clinical Science Laboratory, Inc.) was added, followed by incubation of the plate in the dark for $15 \mathrm{~min}$. The reaction was terminated by adding $2 \mathrm{~N}$ $\mathrm{H}_{2} \mathrm{SO}_{4}$ and then the OD value was measured at $450 \mathrm{~nm}$ wavelength using a microplate ELISA reader (TECAN). The results of the duplicate experiments were averaged and the NGAL levels are expressed as nanograms per milliliter (ng/ml).

The precision and repeatability of the ELISA were evaluated by the coefficient of variation (CV) intra-assay and inter-assay analysis using three concentrations as calibrators $(8625,1078.1,33.69 \mathrm{pg} / \mathrm{mL}$; these were indicated as 
high, medium and low concentrations of the protein). The coefficient of variation $(\mathrm{CV})$ of the average intra-assay and inter-assay CVs were 6.94 and $5.76 \%$, respectively. Additionally, based on the standard curve established by calibrators (standard curve with $\mathrm{R}^{2}=0.99847$ ), NGAL protein levels in clinic samples were measured. All of the ELISAs for measuring NGAL involved three independent repeats. The cut-off value for detection was set by means of negative protein at +3 standard deviations (SD). In general, the detection limit of in-house ELISA was estimated based on the lowest concentration of calibrator with a read higher than cutoff value.

\section{Statistical analysis}

Statistical Product and Service Solutions SPSS $^{\circ} 20$ for Mac) statistical software was used for analysis in this study. Data were first analyzed using the Shapiro-Wilk test to determine the normality. Non-normally distributed data are presented as median and interquartile range (IQR). The Mann-Whitney $U$-test and Kruskal-Wallis Test (post hoc test with Dunn's test) were utilized for the nonparametric analysis to compare the differences between groups or among two groups, respectively. Categorical data are presented as proportions. The chi-square test or Fisher's Exact Test (for any expected cell number $<5$ ) were used to compare these datasets. A $p$ value $p<0.05$ was considered to indicate a significant difference. Spearman's correlation coefficients were applied to determine the associations between different parameters.

\begin{abstract}
Abbreviations
AKI: Acute kidney disease; ANOVA: Analysis of variance; BCIP: 5-bromo-4chloro-3-indolyl phosphate; CKD: Chronic kidney disease; ELISA: Enzymelinked immunosorbent assay; HPF: High power field; IQR: Interquartile ranges; LSD: Least significant difference; MMP-9: Matrix metallopeptidase 9; NBT: Nitro blue tetrazolium; NGAL: Neutrophil gelatinase-associated lipocalin; PBST: Phosphate buffered saline containing 0.1\% Tween-20; RBC: Red blood cell; ROC: Receiver operating characteristics; SDS-PAGE: Sodium dodecyl sulfate polyacrylamide gel electrophoresis; UNCR: Urine NGAL to creatinine ratio; UPC: Urine protein to creatinine ratio; UTI: Urinary tract inflammation; WBC: White blood cell count
\end{abstract}

\section{Acknowledgements}

The authors would like to thank all the doctors at the National Taiwan University veterinary hospital for providing the cases and collecting the samples.

\section{Authors' contributions}

YL, PW and WH designed the experiments, analyzed the data and drafted the manuscript together. PW, YL and HT performed the experiments. WW and PT helped to draft the manuscript. All authors read and approved the final manuscript.

\section{Funding}

This research was financially supported by Ministry of Science and Technology, Taiwan with the number of MOST-105-2311-B-002-027.

\section{Availability of data and materials}

The datasets used and/or analysed during the current study are available from the corresponding author on reasonable request.

\section{Ethics approval and consent to participate}

All the procedures were approved by Institutional Animal Care and Use Committee at National Taiwan University (IACUC Approval No: NTU 103-EL00084).

Consent for publication

Not applicable.

\section{Competing interests}

None of the authors of this paper has a financial or personal relationship with other people or organizations that could inappropriately influence or bias the content of the paper. The authors declare that they have no competing interests.

\section{Author details}

Institute of Veterinary Clinical Science, School of Veterinary Medicine, College of Bio-Resources and Agriculture, National Taiwan University, No. 153, Sec. 3, Keelung Rd, Taipei 106, Taiwan. ${ }^{2}$ Graduate Institute of Microbiology and Public Health, College of Veterinary Medicine, National Chung-Hsing University, 145 Xingda Rd., Taichung 40227, Taiwan.

${ }^{3}$ Department of Veterinary Medicine, School of Veterinary Medicine, National Taiwan University, No.1, Sec.4, Roosevelt Rd, Taipei 106, Taiwan. ${ }^{4}$ National Taiwan University Hospital, No.1, Changde St., Zhongzheng Dist., Taipei 10048, Taiwan. ${ }^{5}$ National Taiwan University Veterinary Hospital, College of Bio-Resources and Agriculture, National Taiwan University, No. 153, Sec. 3, Keelung Rd, Taipei 106, Taiwan.

Received: 15 November 2018 Accepted: 12 August 2019

Published online: 27 August 2019

\section{References}

1. Kjeldsen $\mathrm{L}$, Johnsen $\mathrm{AH}$, Sengelov $\mathrm{H}$, Borregaard N. Isolation and primary structure of NGAL, a novel protein associated with human neutrophil gelatinase. J Biol Chem. 1993;268(14):10425-32.

2. Xu SY, Carlson M, Engstrom A, Garcia R, Peterson CG, Venge P. Purification and characterization of a human neutrophil lipocalin (HNL) from the secondary granules of human neutrophils. Scand J Clin Lab Invest. 1994; 54(5):365-76.

3. Mishra J, Dent C, Tarabishi R, Mitsnefes MM, Ma Q, Kelly C, Ruff SM, Zahedi K, Shao M, Bean J, et al. Neutrophil gelatinase-associated lipocalin (NGAL) as a biomarker for acute renal injury after cardiac surgery. Lancet. 2005; 365(9466):1231-8.

4. Bolignano D, Coppolino G, Campo S, Aloisi C, Nicocia G, Frisina N, Buemi M. Neutrophil gelatinase-associated lipocalin in patients with autosomaldominant polycystic kidney disease. Am J Nephrol. 2007:27(4):373-8.

5. Hsu WL, Lin YS, Hu YY, Wong ML, Lin FY, Lee YJ. Neutrophil gelatinaseassociated lipocalin in dogs with naturally occurring renal diseases. J Vet Intern Med. 2014;28(2):437-42.

6. Liu KD, Yang W, Go AS, Anderson AH, Feldman HI, Fischer MJ, He J, Kallem RR, Kusek JW, Master SR, et al. Urine neutrophil gelatinase-associated lipocalin and risk of cardiovascular disease and death in CKD: results from the chronic renal insufficiency cohort (CRIC) study. Am J Kidney Dis. 2015; 65(2):267-74.

7. Hsu WL, Chiou HC, Tung KC, Belot G, Virilli A, Wong ML, Lin FY, Lee YJ. The different molecular forms of urine neutrophil gelatinase-associated lipocalin present in dogs with urinary diseases. BMC Vet Res. 2014;10:202.

8. Yan L, Borregaard N, Kjeldsen L, Moses MA. The high molecular weight urinary matrix metalloproteinase (MMP) activity is a complex of gelatinase B/MMP-9 and neutrophil gelatinase-associated lipocalin (NGAL). Modulation of MMP-9 activity by NGAL. J Biol Chem. 2001;276(40):37258-65.

9. Cai L, Rubin J, Han W, Venge P, Xu S. The origin of multiple molecular forms in urine of HNL/NGAL. Clin J Am Soc Nephrol. 2010;5(12):2229-35.

10. Lee $Y-J$, Hu Y-Y, Lin Y-S, Chang C-T, Lin F-Y, Wong M-L, Kuo-Hsuan H, Hsu W-L. Urine neutrophil gelatinase-associated lipocalin (NGAL) as a biomarker for acute canine kidney injury. BMC Vet Res. 2012;8(1):248.

11. Segev G, Palm C, LeRoy B, Cowgill LD, Westropp JL. Evaluation of neutrophil gelatinase-associated lipocalin as a marker of kidney injury in dogs. J Vet Intern Med. 2013;27(6):1362-7.

12. Cortellini S, Pelligand L, Syme H, Chang YM, Adamantos S. Neutrophil gelatinase-associated Lipocalin in dogs with sepsis undergoing emergency 
laparotomy: a prospective case-control study. J Vet Intern Med. 2015;29(6): 1595-602.

13. Cobrin AR, Blois SL, Abrams-Ogg AC, Kruth SA, Dewey C, Holowaychuk MK, Gauthier V. Neutrophil gelatinase-associated lipocalin in dogs with chronic kidney disease, carcinoma, lymphoma and endotoxaemia. J Small Anim Pract. 2016;57(6):291-8.

14. Wang IC, Hsu WL, Wu PH, Yin HY, Tsai HJ, Lee YJ. Neutrophil gelatinaseassociated Lipocalin in cats with naturally occurring chronic kidney disease. J Vet Intern Med. 2017;31(1):102-8.

15. Corbacioglu SK, Cevik Y, Akinci E, Uzunosmanoglu H, Dagar S, Safak T, Oncul $\checkmark$, Guvendi M. Value of plasma neutrophil gelatinase-associated lipocalin (NGAL) in distinguishing between acute kidney injury (AKI) and chronic kidney disease (CKD). Turk J Emerg Med. 2017;17(3):85-8.

16. Proverbio D, Spada E, Baggiani L, Bagnagatti De Giorgi G, Ferro E, Martino PA, Perego R. Short communication: relationship between urinary neutrophil gelatinase-associated lipocalin and noninfectious pyuria in dogs. Dis Markers. 2015;2015:387825.

\section{Publisher's Note}

Springer Nature remains neutral with regard to jurisdictional claims in published maps and institutional affiliations.

Ready to submit your research? Choose BMC and benefit from:

- fast, convenient online submission

- thorough peer review by experienced researchers in your field

- rapid publication on acceptance

- support for research data, including large and complex data types

- gold Open Access which fosters wider collaboration and increased citations

- maximum visibility for your research: over $100 \mathrm{M}$ website views per year

At $\mathrm{BMC}$, research is always in progress.

Learn more biomedcentral.com/submissions 